\title{
Case Report on Pinna: An Unusual Site for Verrucous Carcinoma
}

\begin{abstract}
Verrucous carcinoma is an uncommon variant of well-differentiated squamous cell carcinoma and is frequently seen in the oral cavity and larynx having some relation with tobacco chewing, whereas, its presentation over the ear is a rare entity. Here, we report the case of a 70 -year-old female patient with complaints of cauliflower-like mass over her left pinna which was managed by doing an excisional biopsy and diagnosed as verrucous carcinoma on histopathology.

Keywords: Carcinoma, pinna, unusual site, verrucous
\end{abstract}

\section{Introduction}

Verrucous carcinoma is a variant of well-differentiated squamous cell carcinoma (SCC) with specific clinical, pathological, and behavioral peculiarities to justify its being regarded as a specific tumor entity. ${ }^{[1]}$ It develops most frequently in the mucous membranes of the oral cavity and larynx. The development of this tumor in the ear is particularly rare. There are only 16 cases reported of verrucous carcinoma affecting the ear in the literature. ${ }^{[2]}$ It presents as slow-growing fungating mass or irregular elevated plaque. Usually, verrucous carcinomas develop without sex-related predominance in individuals aged $50-80$ years. A full-thickness biopsy is necessary to ensure the correct diagnosis. The treatment of choice is surgical excision. We are reporting a case of verrucous carcinoma over the helix of the left pinna for which surgical excision was preferred as the treatment of choice. ${ }^{[3]}$

\section{Case Report}

A 70-year-old female patient, previously farmer by occupation, presented with complaints of a fungating growth over her left pinna for 2 years. It was insidious in onset and was gradually progressive in size. Initially, it was smaller in size and had a papular presentation which gradually progressed to presenting condition which had cauliflower-like shape and approximately 3 $\mathrm{cm} \times 3 \mathrm{~cm} \times 2 \mathrm{~cm}$ [Figure 1]. The patient has had complaints of itching over the lesion

This is an open access journal, and articles are distributed under the terms of the Creative Commons Attribution-NonCommercial-ShareAlike 4.0 License, which allows others to remix, tweak, and build upon the work non-commercially, as long as appropriate credit is given and the new creations are licensed under the identical terms.

For reprints contact: WKHLRPMedknow_reprints@wolterskluwer.com since the beginning but developed pain over the lesion during the past 1 month. The pain was dull aching, continuous, aggravated on touch, and relieved with medication. There is no history of trauma or burn injury or insect bite at the site of the lesion and no history of any other similar lesions in the body. She is a known hypertensive for which she is on medication and has addiction to tobacco use orally in the form of kharra (local preparation of tobacco used orally) for the past 30 years.

The above mentioned growth was managed by doing an excisional biopsy along with the underlying helical cartilage and was sent for histopathology examination, and the local site was closed with primary suturing [Figure 2].

Histopathology examination showed the image of a verrucous carcinoma (showing rete ridges with bulldozing pattern and hyperkeratosis) without exceeding the resection limits [Figure 3].

\section{Discussion}

Most of the malignancies that involve the external ear are cutaneous malignancies, of which basal cell carcinoma (BCC), SCC, and melanoma constitute the maximum numbers. Although skin cancer can occur in all age groups, the vast majority of patients presenting with cutaneous carcinoma are older. The mean age of patients with BCC or SCC is near 70 years.

The incidence of skin cancer is much lower in darker-skinned ethnic groups than in Caucasians, who represent $95 \%$ of patients. BCC represents $65 \%-85 \%$ of head and neck cutaneous malignancies and a similar

How to cite this article: Khan S, Akulwad SB, Sonone J. Case report on pinna: An unusual site for verrucous carcinoma. Indian J Med Paediatr Oncol 2020;41:906-8.
Sidra Khan, Shivkumar Balaji Akulwad, Jayant Sonone

Department of ENT, MGIMS, Sevagram, Wardha, Maharashtra, India

Submitted: 19-Jan-2020 Revised: 17-Jul-2020 Accepted: 28-Jul-2020 Published: 31-Dec-2020

Address for correspondence: Dr. Sidra Khan, JN Girls Hostel, MGIMS, Sevagram, Wardha - 442 102, Maharashtra, India.

E-mail:sidra.khan1692@gmail. com

Access this article online

Website: www.ijmpo.org

DOI: 10.4103/ijmpo.ijmpo_19_20

Quick Response Code:

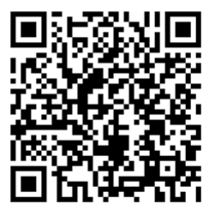




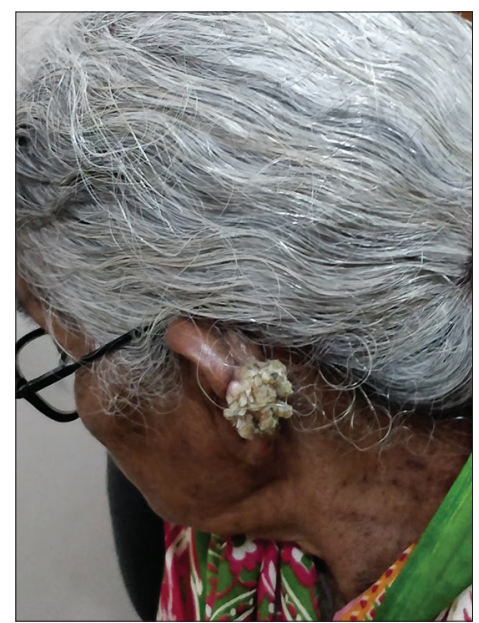

Figure 1: Cauliflower-like growth presents on the helix of the left pinna

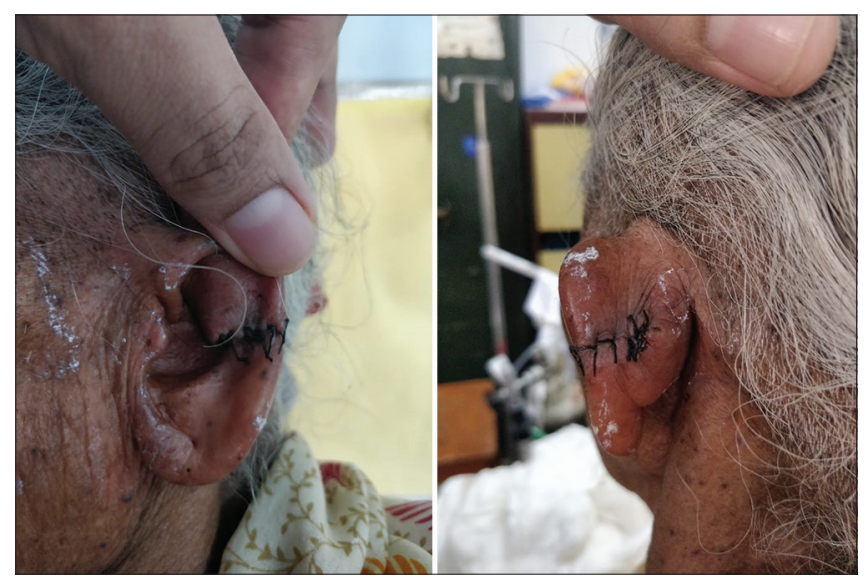

Figure 2: Postoperative images after excisional biopsy of verrucous growth over the helix of the left pinna

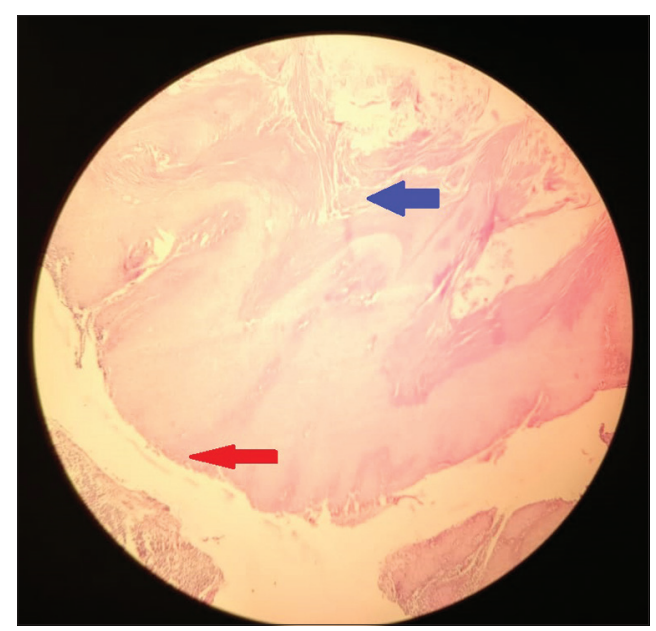

Figure 3: Histopathological picture of verrucous carcinoma showing hyperkeratosis (blue arrow) and rete ridges (red arrow)

proportion of auricular carcinomas. SCC is the second most common, followed by melanoma. ${ }^{[4]}$

The patient who we report here is a 70-year-old female having fungating exophytic growth over her left auricle of the pinna [Figure 1]. There is differences in the distribution of BCCs versus SCCs. SCC arises most frequently on the external ear and upper face, whereas BCC occurs most commonly in the midface, followed by the ear. When these lesions appear on the auricle, $72 \%$ of the BCCs and $61 \%$ of the SCCs are confined to a single subsite of the ear. BCC is found primarily on the posterior surface of the auricle, followed by the preauricular and then the retroauricular areas. SCC occurs in order of decreasing frequency on the helical rim, antihelix and triangular fossa, and posterior pinna and then the concha, lobule, or tragus. ${ }^{[4]}$

Sun exposure is the number one risk factor for the development of cutaneous malignancies. The correlation between sun exposure and SCC is stronger than that with $\mathrm{BCC}$. The sun is the primary source of human ultraviolet radiation and the radiation ranges in wavelength from 200 to $400 \mathrm{~nm}$ and has been divided into three groups. Ultraviolet A rays (320-400 nm) make up the majority of the ultraviolet radiation reaching the earth and therefore produce most of the damage to our skin. ${ }^{[4]}$

Verrucous carcinoma is a variant of well-differentiated SCC with specific clinical, pathological, and behavioral peculiarities to justify its being regarded as a specific tumor entity. ${ }^{[1]}$ Various names are used in the literature to describe this entity, including Ackerman's tumor, Buschke-Lowenstein tumor, fibroid oral papillomatosis, epithelioma cuniculatum, snuff dipper's cancer, and carcinoma cuniculatum. ${ }^{[5]}$ It develops most frequently in the mucous membranes of the oral cavity and larynx. The development of this tumor over and in the ear has been reported in 16 cases. ${ }^{[2]}$

Risk factors for verrucous carcinoma enumerated are smoking, human papillomavirus $6,11,16$, and 18, infections, trauma, poor hygiene, minor emissions of irradiations, immunodeficiency, exposure to sunlight and old scars, tobacco chewing, etc. ${ }^{[3]}$ With relation to the anatomic position of the auricle, sunlight exposure can be attributed as the key risk factor.

The patient reported in our case was a farmer by occupation, and India being a tropical country, sunlight exposure maybe considered as the contributing factor in the development of verrucous carcinoma. It presents as slow-growing fungating mass or irregular elevated plaque, as in our case, it was a slow-growing fungating mass [Figure 1]. It is locally invasive and nonmetastasizing.

The treatment of choice is surgical excision because verrucous carcinomas are less radiosensitive than typical SCCs. ${ }^{[3]}$ The patient reported in our case was also treated on similar lines of excisional biopsy with the removal of underlying cartilage, and the diagnosis was confirmed on histopathological examination [Figure 2].

Morbidity in verrucous carcinoma is due to local aggressiveness of the tumor (consisting in the skin and soft-tissue destruction) and perineural, muscle, and bone invasion. Metastases in verrucous carcinoma are so rare that the mortality is more often due to local invasion. ${ }^{[6]}$ 
Verrucous carcinoma has varied clinical presentation. It is made up of bland epithelial cells that tend to heap up on the surface. The rate of malignant transformation to SCC remains high. Although it is a locally aggressive lesion with rare nodal metastases, the decision to do a conservative neck dissection is reserved for the hybrid type of verrucous carcinoma. ${ }^{[7]}$ It appears usually on a previous injury, often affecting the buccal mucosa. In patients with suspicious lesions, more than one biopsy to diagnose a verrucous carcinoma may be needed. No cervical lymph node involvement was seen in the study done by Candau-Alvarez et al. Local resection with at least $5 \mathrm{~mm}$ of histological margin, without prophylactic neck dissection allows local control of the disease. ${ }^{[8]}$

Verrucous carcinoma can also transform into SCC, which is dependent on the marked reduction of membranous transforming growth factor beta receptor 2 (TBR-II) and their predominant cytoplasmic location which further diminishes transforming growth factor-beta growth inhibition which may cause the transformation. ${ }^{[9]}$

As reported in the study done by Singh et al., they had come across a verrucous carcinoma over the helix of the pinna quite similar to the case presented by us, but there they had a correlation of the lesion with burn injury ${ }^{[3]}$ over the same site some years back, whereas in our case, we did not see any such correlation, but the patient in our case being a farmer had a higher rate of exposure to sunlight which may had a role in the development of the tumor over the auricle.

Another study done by Costache et al. from Romania was the verrucous carcinoma of the right auricle in an 86-year-old female, which was consistent with our report, but there they had also come across a smaller nodular growth, whereas our reported lesion was nodular to start with but later turned into a fungating one. ${ }^{[6]}$

The study done by Proops et al. in England had reported verrucous carcinoma in the middle ear for which mastoidectomy was performed, and the final diagnosis was given after histopathological correlation. ${ }^{[10]}$

The best treatment modality considered in case of verrucous carcinoma is surgical excision with up to $5-10 \mathrm{~mm}$ margin, and as this tumor rarely presents with nodal metastasis, neck dissection is considered only in some hybrid cases of verrucous carcinoma. As compared with SCC, radiotherapy does not play much role in the treatment of verrucous carcinoma. In fact, some studies have shown radiotherapy to induce anaplastic changes if given in cases of verrucous carcinoma. ${ }^{[1]}$ The study done by Mohan et al. also reports that verrucous carcinoma which is treated with adjuvant radiotherapy has a worse overall survival and disease-specific survival as compared to both verrucous carcinoma treated with surgery alone and SCC treated with surgery and adjuvant radiotherapy. ${ }^{[12]}$

\section{Conclusion}

The variant of SCC known as verrucous carcinoma is commonly found in the oral cavity and in the larynx, whereas the ear is a rare site for the same. The diagnosis of verrucous carcinoma should be considered in case of any mass or lesion over the ear auricle, which should be treated with surgical excision, and the diagnosis is made by histopathological examination.

Radiotherapy has no role in the management of verrucous carcinoma, and morbidity in these cases is only due to local aggressiveness of the tumor and not due to metastasis.

\section{Declaration of patient consent}

The authors certify that they have obtained all appropriate patient consent forms. In the form, the patient has given her consent for her images and other clinical information to be reported in the journal. The patient understands that name and initials will not be published and due efforts will be made to conceal the identity, but anonymity cannot be guaranteed.

\section{Financial support and sponsorship}

Nil.

\section{Conflicts of interest}

There are no conflicts of interest.

\section{References}

1. Ackerman LV. Verrucous carcinoma of the oral cavity. Surgery 1948;23:670-8.

2. Nam SJ, Yang CJ, Chung JW. A case of squamous cell carcinoma in the external auditory canal previously treated for verrucous carcinoma. J Audiol Otol 2016;20:183-6.

3. Singh A, Verma N, Sachan B, Negi V. Verrucous carcinoma on the helix of pinna. N Am J Med Sci 2013;5:153-4.

4. Keith A. Casper, Myles Pensak Surgery for Cancer of External Ear. Surgery of the Ear. $6^{\text {th }}$ ed. Glasscock-Shambaugh, Shelton; 2010. p. 399-400.

5. Schwartz RA. Verrucous carcinoma of the skin and mucosa. J Am Acad Dermatol 1995;32:1-21.

6. Costache M, Desa LT, Mitrache LE, Pătraşcu OM, Dumitru A, Costache D, et al. Cutaneous verrucous carcinoma - report of three cases. Rom J Morphol Embryol 2014:55:383-8.

7. Holenarasipur SN, Nagaraj T, Batlahalli MV, Tathanahalli YL. Atypical variation of oral verrucous carcinoma: A comprehensive case report. J Indian Acad Oral Med Radiol 2016;28:70-3.

8. Candau-Alvarez A, Dean-Ferrer A, Alamillos-Granados FJ, Heredero-Jung S, García-García B, Ruiz-Masera JJ, et al. Verrucous carcinoma of the oral mucosa: An epidemiological and follow-up study of patients treated with surgery in 5 last years. Med Oral Patol Oral Cir Bucal 2014;19:e506-11.

9. Anderson M, Muro-Cacho C, Cordero J, Livingston S, MuñozAntonia T. Transforming growth factor beta receptors in verrucous and squamous cell carcinoma. Arch Otolaryngol Head Neck Surg 1999;125:849-54.

10. Hawke WM, van Nostrand AW, Harwood AR, Lunan M. Verrucous carcinoma of the ear. Ann Otol Rhinol Laryngol 1984;93:385-88.

11. Jyothirmayi R, Sankaranarayanan R, Varghese C, Jacob R, Nair MK. Radiotherapy in the treatment of verrucous carcinoma of the oral cavity. Oral Oncol 1997;33:124-8.

12. Mohan S, Pai SI, Bhattacharyya N. Adjuvant radiotherapy is not supported in patients with verrucous carcinoma of the oral cavity. Laryngoscope 2017;127:1334-8. 